\title{
Simulation of Atmospheric Pressure Effects on Particle Densities Measured by GRAPES-3
}

M. Zuberi ${ }^{a b}$, S. Ahmad ${ }^{a c}$, M. Chakraborty ${ }^{a b}$, A. Chandra ${ }^{a b}$, S.R. Dugad ${ }^{a b}$, S.K. Gupta ${ }^{a b}$, B. Hariharan ${ }^{a b}$, Y. Hayashi ${ }^{a d}$, P. Jagadeesan ${ }^{a b}$, A. Jain ${ }^{a b}$, P. Jain ${ }^{a g}$, V.B. Jhansi ${ }^{a b}$, S. Kawakami ${ }^{a d}$, H. Kojima ${ }^{a e}$, S. Mahapatra ${ }^{a h}$, P.K. Mohanty ${ }^{a b}$, S.D. Morris ${ }^{a b}$, Y. Muraki ${ }^{a i}$, P.K. Nayak ${ }^{a b}$, A. Oshima ${ }^{a f}$, D. Pattanaik ${ }^{a b}$, P.S. Rakshe ${ }^{a b}$, K. Ramesh ${ }^{a b}$, B.S. Rao ${ }^{a b}$, L.V. Reddy ${ }^{a b}$, S. Sharma ${ }^{a b}$, S. Shibata ${ }^{a f}$, K. Tanaka ${ }^{a j}$, F. Varsi ${ }^{a g}$

${ }^{a}$ The GRAPES-3 Experiment, Cosmic Ray Laboratory, Raj Bhavan, Ooty 643001, India

${ }^{b}$ Tata Institute of Fundamental Research, Mumbai 400005, India

${ }^{c}$ Aligarh Muslim Univeristy, Aligarh 202002, India

${ }^{d}$ Graduate School of Science, Osaka City University, Osaka 558-8585, Japan

${ }^{e}$ Faculty of Engineering, Aichi Insitute of Technology, Toyota City, Aichi 470-0392, Japan

${ }^{f}$ College of Engineering, Chubu University, Kasugai, Aichi 487-8501, Japan

${ }^{g}$ Indian Institute of Technology Kanpur, Kanpur 208016, India

${ }^{h}$ Utkal University, Bhuvaneshwar 751004, India

${ }^{i}$ Institute for Space-Earth Environmental Research, Nagoya University, Nagoya 464-8601, Japan

${ }^{j}$ Graduate School of Information Sciences, Hiroshima City University, Hiroshima 731-3194, Japan

E-mail: meeran.zuberihepegmail.com

The GRAPES-3 is a high density extensive air shower (EAS) array located at Ooty India. The array is presently operating with 400 plastic scintillation detectors ( $1 \mathrm{~m}^{2}$ area each) with the interdetector separation of $8 \mathrm{~m}$ spread over $25,000 \mathrm{~m}^{2}$ and a large area $\left(560 \mathrm{~m}^{2}\right)$ tracking muon detector.The variation in atmospheric pressure can significantly affect the observed cosmic ray flux measured by these detectors. We have simulated this effect by using CORSIKA package after folding the detector response using GEANT4. The results of this work will be discussed during the conference.

36th International Cosmic Ray Conference -ICRC2019-

July 24th - August 1st, 2019

Madison, WI, U.S.A.

\footnotetext{
* Speaker.
} 


\section{Introduction}

Cosmic rays are the relativistic charged particles impinging on the Earth's atmosphere from outer-space and develop the extensive air showers (EAS) by interacting with the atmosphere's gaseous nuclei. As the atmosphere acts as a medium where the EAS develops, its variability affects the lateral and longitudinal development of the shower. Atmospheric pressure is the major property of the atmosphere which mostly affects the EAS and define as the force per unit area exerted by an atmospheric column. An increase (or decrease) of the atmospheric pressure corresponds to and increased (or decreased) amount of matter traversed by shower particles, which affects the stage of longitudinal development of the shower when it reaches the ground. The cosmic ray intensity variations due to modulation in pressure is known from the time of cosmic rays discovery. There have been several attempts to study this effect by many ground based detectors and by now we have a fairly good understanding of this phenomena [1, 2, 3]. There are many EAS simulation packages amoung them CORSIKA [4] is widely used by cosmic ray community. It has several low and high energy interaction models which can be used in different combinations to simulate the EAS. However, as discussed earlier, atmospheric conditions for ground-based experiments are very crucial for doing any precision study. We have attempted to simulate the atmospheric pressure effect by modifying the atmospheric parameters. We have also studied the effect of different interaction model combinations to check their reliability by comparing the simulated results with observed pressure coefficient obtained by using GRAPES-3 EAS data.

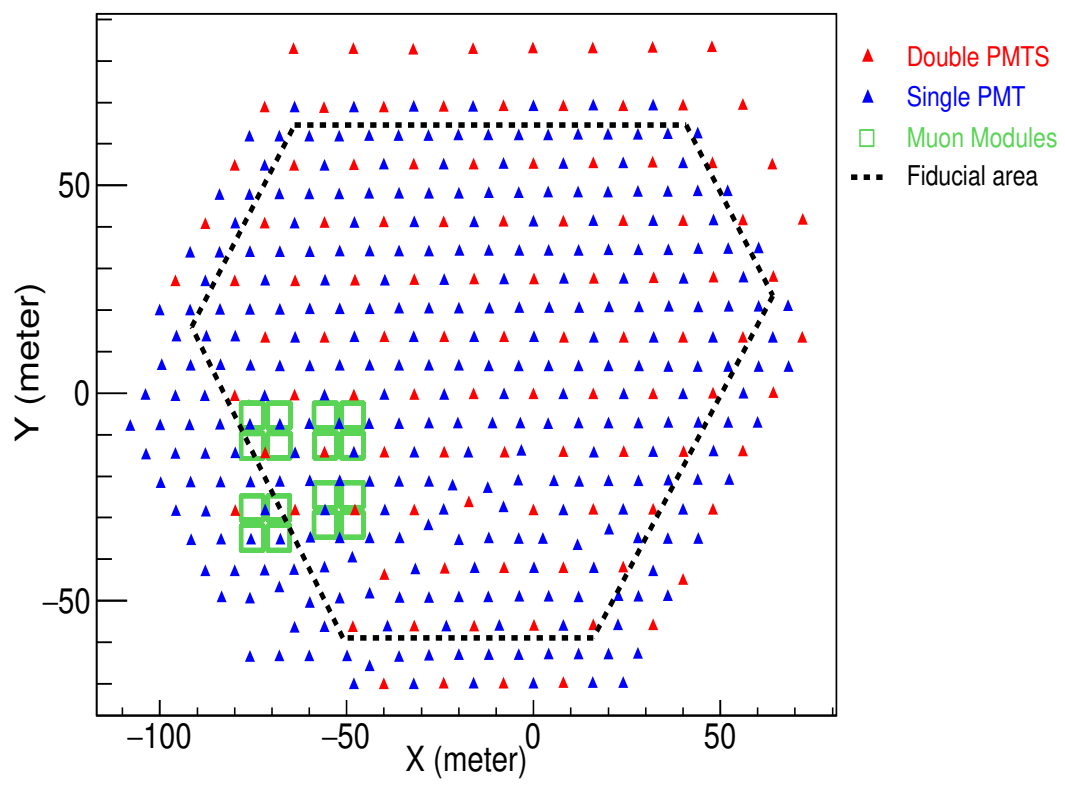

Figure 1: Schematic layout for GRAPES-3 shower array

The GRAPES-3 (Gamma Ray Astronomy at PeV EnergieS - phase 3) is a high density EAS array, designed for precision studies of cosmic rays energy spectrum, composition, acceleration in atmospheric and various solar induced phenomenon. It is located at Ooty $\left(2.2 \mathrm{~km}\right.$ altitude, $11.4^{\circ} \mathrm{N}$, $76.7^{\circ} \mathrm{E}$ ) in southern part of India. Presently there are 400 plastic scintillation detectors deployed 
on a hexagonal pattern with the inter-detector separation of $8 \mathrm{~m}$ as shown in Fig. 1. In addition to that, Fig. 1 also shows sixteen squares in the north-west region. Each of these squares represents a tracking muon telescope ( $35 \mathrm{~m}^{2}$ area) with $1 \mathrm{GeV}$ energy threshold for vertical muons having the total area of $560 \mathrm{~m}^{2}$. The detailed description of GRAPES-3 scintillation detectors and muon telescope is discussed elsewhere [5, 6].

\section{Pressure Effect on Particle Density}

The high statistical EAS data recorded by 400 plastic scintillation detectors were used to calculate pressure coefficients for individual detectors. Due to usage of photo multiplier tube for photons conversion to the electrical signal in plastic scintillation detectors, hourly particle density shows a strong temperature dependence. A fast Fourier transform (FFT) technique was used to segregate the temperature and pressure effects. Further study reveals the presence of delay between temperature and particle densities for all detectors. After taking into account of time delay, the particle densities were corrected for the temperature effect then they were correlated with pressure which shows very high degree of correlation. The mean pressure coefficients were calculated for all scintillation detectors which is found to be $(-0.68 \pm 0.01) \% \mathrm{hPa}^{-1}$ with $0.1 \% \mathrm{hPa}^{-1}$ rms as shown in Fig. 2, very similar to the earlier reports [7, 8]. However, there is no lag observed between PD and pressure which is expected because pressure effect is independent of detector properties and therefore, all the detectors pressure coefficients are observed to be very similar which is reflected by narrow spread in coefficients. More technical details of atmospheric effects on particle densities can be found elsewhere [9].

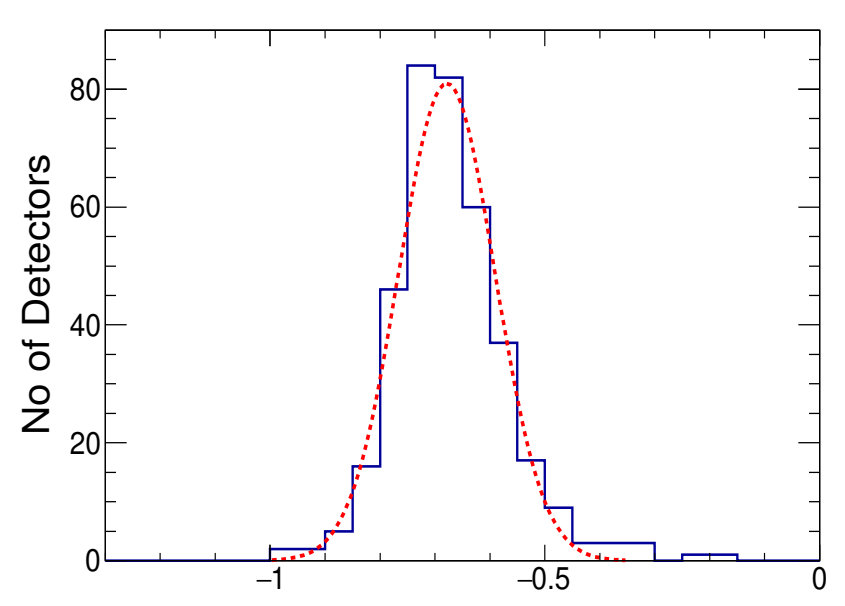

Figure 2: Distribution of pressure coefficients of all the detectors for year 2014. The average value is found to be $(-0.68 \pm 0.01) \% h \mathrm{~Pa}^{-1}$ with $0.1 \% h \mathrm{~Pa}^{-1} \mathrm{rms}$.

\section{The Monte Carlo Simulations}

The pressure dependence of cosmic rays simulation for GRAPES-3 experiment was done in 
two steps. Firstly, by using CORSIKA package, cosmic rays showers were generated for different interaction models and then these showers were used as an input of GEANT4 simulation package for obtaining the effect of pressure changes in GRAPES-3 EAS array. CORSIKA (COsmic Ray SImulation for KAscade) is a detailed Monte Carlo program developed to study the development and properties of EAS in the atmosphere [4]. We have simulated $35 \times 10^{4}$ proton initiated showers ( $10^{13}$ to $10^{16}$ energy range) in GRAPES-3 fiducial area (as shown in 1 by black dashed line) with the nine possible combinations of high energy (SIBYLL [10], QGSJETII [11] and EPOS [12]) and low energy (FLUKA [13], GHEISHA [14] and URQMD [15]) hadronic interaction models. In CORSIKA the atmospheric density variation with altitude is modeled by 5 layers. The density of the lower 4 layers follows and exponential dependence on the altitude which can be expressed in terms of mass overburden $T(h)$ of the atmosphere and height $h$ as:

$$
T(h)=a_{i}+b_{i} \cdot e^{-h / c_{i}}
$$

where i runs from layer 1 to 4 . However, for the 5th layer, the mass overburden decreases linearly with height as:

$$
T(h)=a_{5}-b_{5} \cdot h / c_{5}
$$

Here a, b and c are the parameters taken from "Central European atmosphere" (ATMOD5) atmospheric model which gives the closest value of pressure at Ooty altitude. Later, the parameter ' $\mathrm{b}$ ' was modified by changing its value $\pm 3 \%$ in steps of $1 \%$. Fig. 3 shows the variation of atmospheric density for unmodified atmosphere (by dashed red line), -3\% change (by solid blue) and 3\% in parameter 'b' (by black long dashed line). Expectedly, all three plots showed exponential behavior with $-3 \%$ has highest and 3\% has lowest atmospheric density for a given height. In all seven atmospheric conditions proton initiated showers were generated.

After generating the showers in modified atmosphere, they were randomly thrown over fiducial area of GRAPES-3 array (as shown by dashed black line in Fig. 1). The response of plastic scintillation detectors have been studied by using simulated showers and GEANT4 package. For every set, the sum of observed particles in all detectors were calculated and then normalized to their mean value.

\section{Results and Discussions}

Earth's atmosphere plays a vital role in indirect cosmic rays measurement studies. Any periodic changes in atmospheric conditions can modulate observed comic rays flux. The atmospheric pressure effect on cosmic rays is a well known phenomena and have been studied in past $[1,2,3]$. GRAPES-3 experiment has also studied the pressure effect of particle densities. The observed average pressure coefficient for all the plastic scintillation detector is $(-0.68 \pm 0.01) \% h \mathrm{~Pa}^{-1}$ with $0.1 \% \mathrm{hPa}^{-1} \mathrm{rms}$. Furthermore, we have attempted to simulate this effect by modifying the atmospheric parameters of CORSIKA and generated the proton initiated showers for different combinations of high and low energy hadronic interaction models as discussed earlier. Fig. 4 shows 


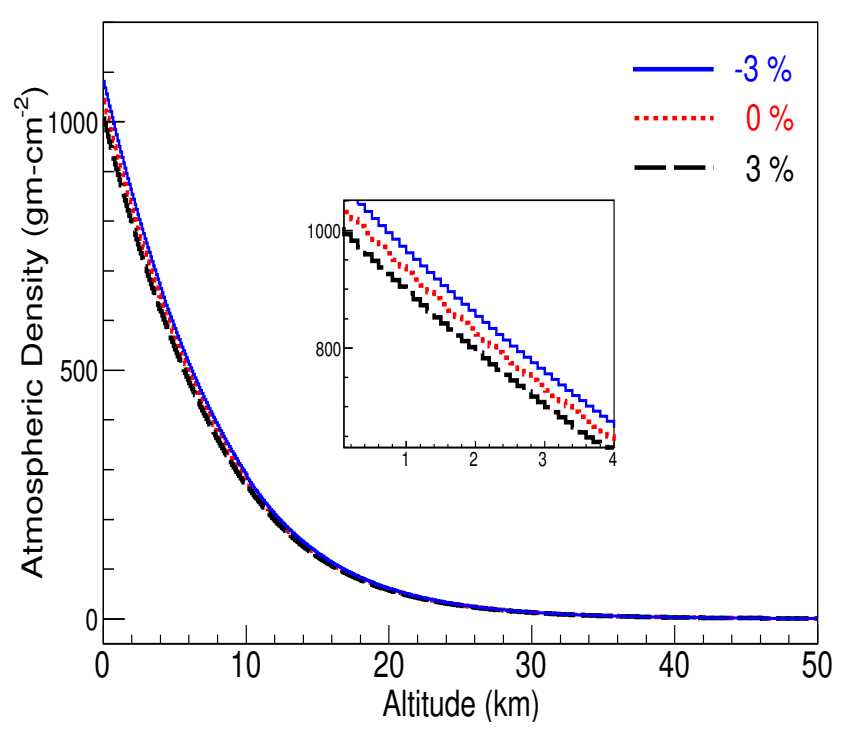

Figure 3: Atmospheric density variation as a function of altitude from 0 to $50 \mathrm{~km}$ for different atmospheric conditions. The inset plot shows the zoomed view from 0 to $4 \mathrm{~km}$.

\begin{tabular}{|c|c|c|c|}
\hline $\begin{array}{c}\text { High/Low } \\
\text { Energy Models }\end{array}$ & GHEISHA & FLUKA & URQMD \\
\hline SIBYLL & $-0.45 \pm 0.01$ & $-0.43 \pm 0.01$ & $-0.45 \pm 0.01$ \\
\hline QGSJETII & $-0.49 \pm 0.01$ & $-0.47 \pm 0.02$ & $-0.50 \pm 0.02$ \\
\hline EPOS & $-0.44 \pm 0.01$ & $-0.42 \pm 0.01$ & $-0.45 \pm 0.01$ \\
\hline
\end{tabular}

Table 1: Regression coefficients for different combinations of high and low energy interaction models. Columns and

the ratio between SIBYLL-FLUKA combination to the other high and low energy hadronic interaction model combinations for each pressure value which clearly shows the model dependence. Fig. 4 also shows that for a given high energy model the ratio for different low energy models are comparable. However, for high energy models the difference in ratios are clearly visible. The combination of low energy models with QGSJETII shows the least value of ratios, with EPOS it shows moderately higher values then QGSJETII and with SIBYLL combined with two other low energy models named as GHEISHA and URQMD shows the highest values of ratios $(\sim 1)$ as compared to the other high and low energy model combinations.

The sum of observed particle were calculated which were then normalized with respect to the mean value for a given model as shown in Fig. 5. Afterwards, the regression coefficients were obtained for every high and low energy model combination as tabulated in Table 1. The regression coefficients for SIBYLL and EPOS high energy model combination with low energy models shows the comparable values. However, for QGSJETII, the regression coefficients with other low energy models are found to be systematically higher as compare to other two high energy models. 


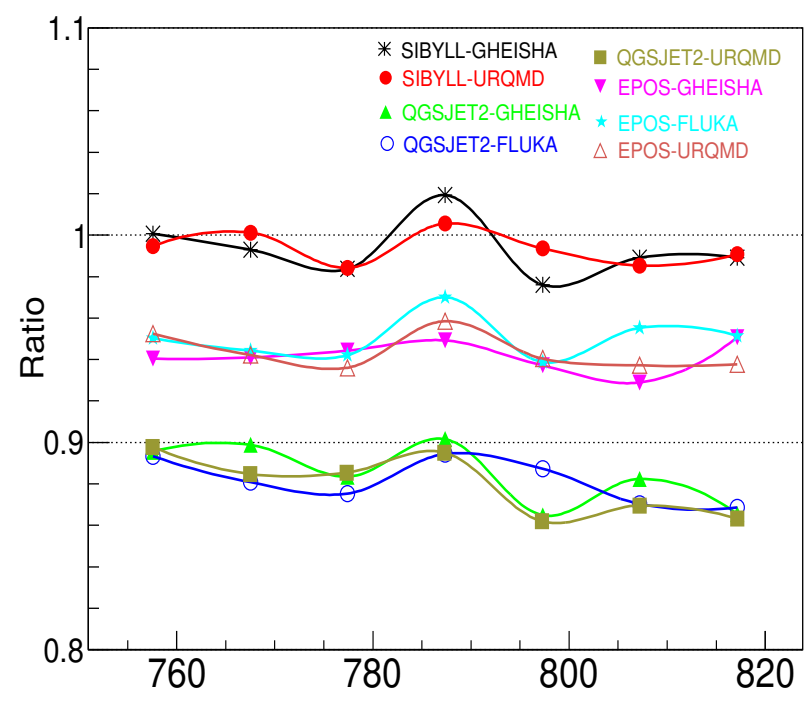

Figure 4: Ratio between SIBYLL-FLUKA combination to the other high and low energy hadronic interaction model combinations for different pressure values.

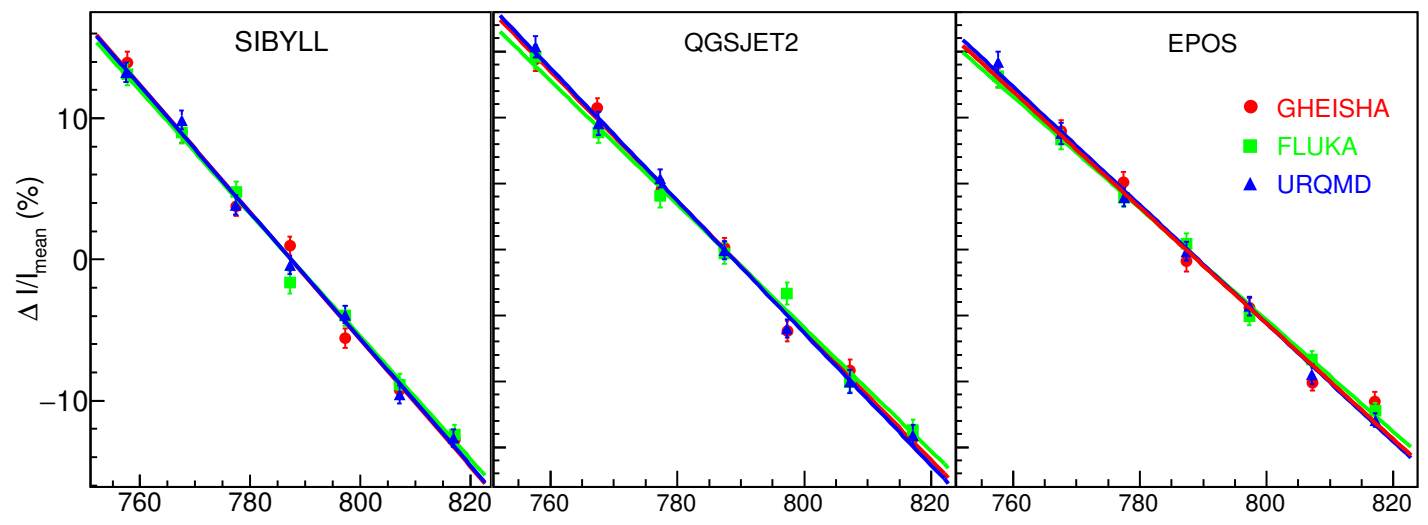

Figure 5: Mean number of particles at different pressure for different high and low energy model combinations.

\section{Acknowledgement}

We thank D.B. Arjunan, G.P. Francis, V. Jeyakumar, S. Kingston, K. Manjunath, S. Murugapandian, S. Pandurangan, B. Rajesh, K. Ramadass, V. Santoshkumar, M.S. Shareef, C. Shobana, R. Sureshkumar and other colleagues for their help in running and maintenance of the GRAPES-3 experiment. 


\section{References}

[1] Wulf, Th., Beobachtungen Ãijber Strahlung hoher Durchdringungsfãd'higkeit auf dem Eiffelturm, Phys. Z. 11, 811-813 (1910).

[2] Myssowsky, L., Tuwim, L., UnregelmÃd'ssige IntensitÃd'tschwankungen der HÃúhenstrahlung in geringer SeehÃúhe, Phys. Z. 39, 146-150 (1926).

[3] Adamson, P., et al., Observation of muon intensity variations by season with the MINOS far detector, Phys. Rev., D(81), 012001, (2010).

[4] https://www.ikp.kit.edu/corsika/.

[5] S.K. Gupta, et al., Nuclear Instruments and Methods in Physics Research A, 540, 311 (2005).

[6] Y. Hayashi, et al., Nuclear Instruments and Methods in Physics Research A, vol. 545, 643 (2005).

[7] Daudin, A. and Daudin, J., Effects atmospheriques sur les gerbes d'Auger, Journal of Atmospheric and Terrestrial Physics, vol. 3, 245 (1953).

[8] Lindgren, S., On the pressure dependence of the cosmic ray intensity recorded by the standard neutron monitor, Tellus, vol. 14, 44-48 (1962).

[9] https://pos.sissa.it/301/302/pdf.

[10] E. J. Ahn, R. Engel, T. K. Gaisser, P. Lipari, and T. Stanev,Phys. Rev. D80, 094003 (2009).

[11] N. N. Kalmykov, S. S. Ostapchenko, and A. I. Pavlov,Nucl.Phys. B, Proc. Suppl.52B, 17 (1997).

[12] T. Pierog, I. Karpenko, J. M. Katzy, E. Yatsenko, and K.Werner,Phys. Rev. C92, 034906 (2015).

[13] T. T. Bhlen, F. Cerutti, M. P. W. Chin, A. Fass, A. Ferrari,P. G. Ortega, A. Mairani, P. R. Sala, G. Smirnov, and V.Vlachoudis,Nucl. Data Sheets120, 211 (2014).

[14] http://cds.cern.ch/record/162911/files/CM-P00055931.pdf

[15] http://urqmd.org. 